\title{
Reversible deficits of functional brain connectivity in patients with carotid artery stenosis
}

\section{Background}

- Internal carotid artery (ICA) stenosis is a well-known risk factor for ischemic stroke. Moreover, even in the absence of stroke, asymptomatic carotid stenosis has been associated with cognitive impairment (Balestrini et al. 2013).

- Primary fMRI studies have shown changes of functional brain connectivity in patients with stenosis (Cheng et al. 2012)

- It has been suggested that disturbed functional brain connectivity might underlie cognitive impairment in asymptomatic carotid stenosis in the absence of structural brain damage.

\section{Objectives}

I. How does functional brain connectivity change with chronic cerebral hypoperfusion?

II. Are connectivity changes reversible after revascularization ?

\section{Conclusion}

We characterized differences of functional brain connectivity in patients with asymptomatic ICA stenosis.

Main findings

I. Functional brain connectivity decreases in patients with chronic hypoperfusion.

II. Connectivity changes are reversible after revascularization.

Hence, even in patients with asymptomatic stenosis, we found disturbances in the underlying brain network that might be the cause for cognitive changes. These network changes, however, are reversible after treatment of the stenosis, giving rise to the question whether patients with an asymptomatic stenosis should be offered treatment, even in the absence of stroke or transient ischemic attacks.

Electrophysiological data were recorded from 12 patients with asymptomatic ICA stenosis pre- and post revascularization (carotid endarterectomy or carotid artery stenting ) as well as from 23 age-eligible controls.

\section{Results}

\section{Connectivity decreases in patients with stenosis and increases after revascularization}

controls

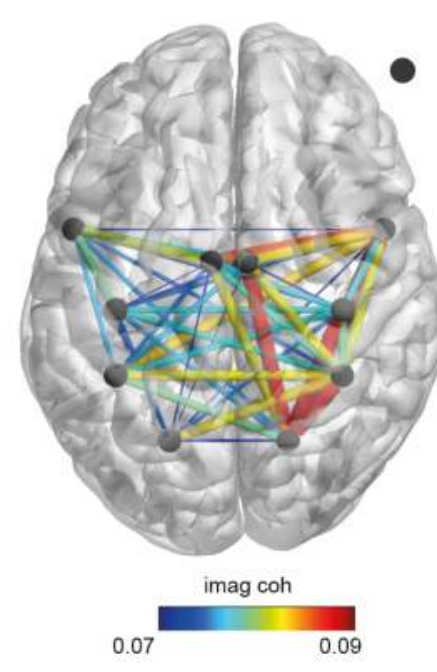

patients pre-intervention

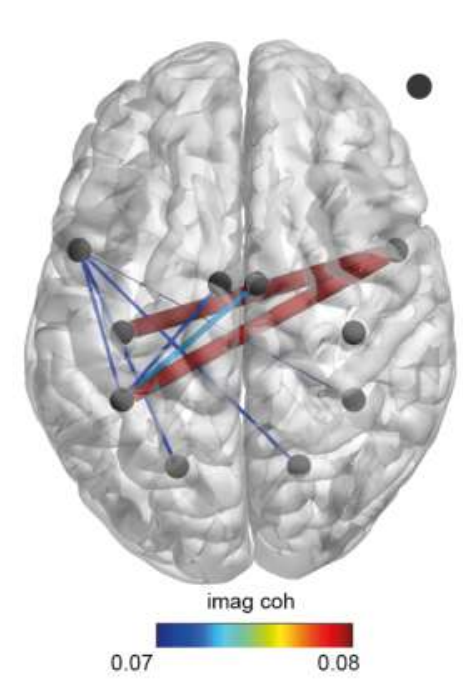

Statistical model results. Linear mixed model with the interaction of group (1 controls, 2 patients pre-intervention, 3 patients post-intervention) and side (-1 non-stenotic side, 0 interhemispheric, 1 stenotic side). Post-hoc testing revealed a significant decrease of connectivity in patients with stenosis compared to controls as well as a significant increase in patients after intervention compared to before intervention. Patients after intervention, however, did not statistically differ from controls anymore. Asterisks mark significance in post-hoc testing.

patients

post-intervention

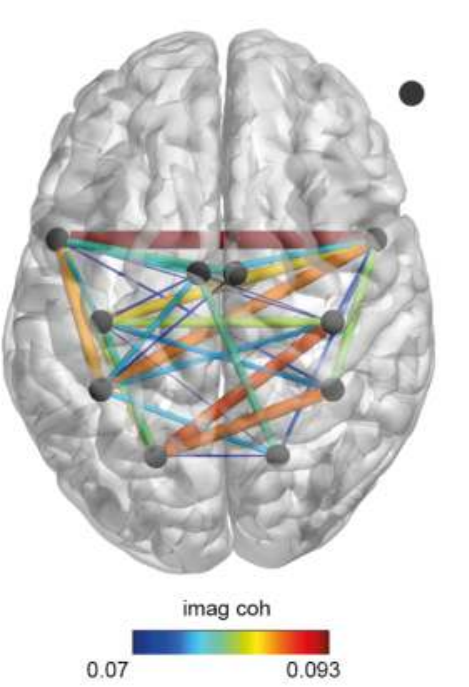

Imaginary coherence of all three groups. The mean alpha imaginary coherence across participants above a threshold of 0.07 is plotted for each group. Warm colors and larger size of connections represent a greater imaginary coherence, whereas cold colors and smaller size represent less imaginary coherence. Dot marks the side of stenosis.

Statistical evaluation of connectivity changes using a linear mixed model

controls vs patients pre-intervention

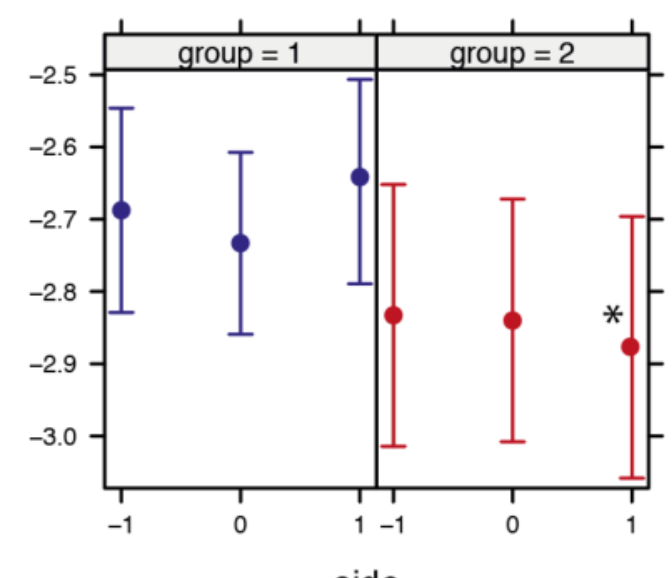
patients pre-intervention vs patients post-intervention

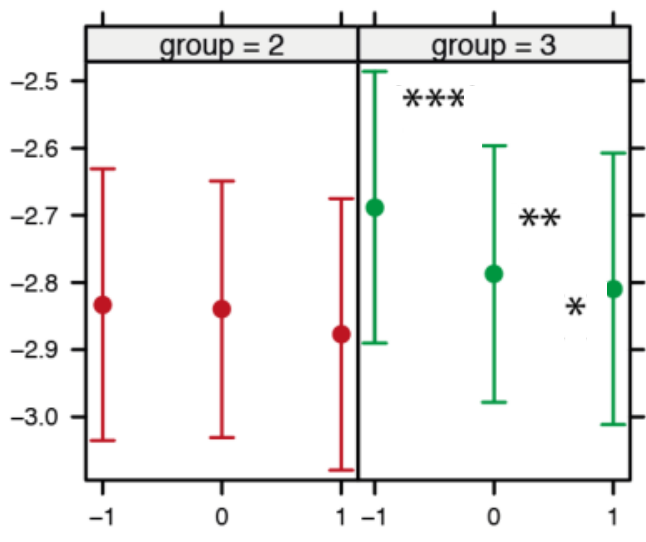

side controls vs patients post-intervention

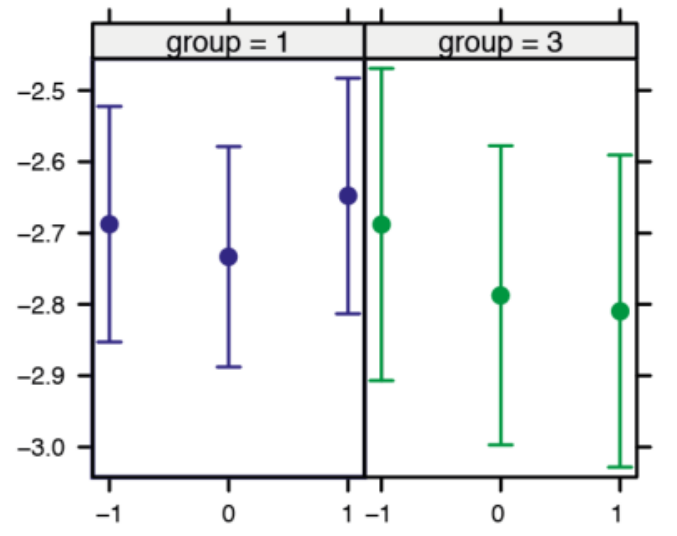

side

\section{Methods}

Participants

- group1: 23 age-eligible controls

- group2: 12 patients with high grade carotid artery stenosis

group3: 12 patients (as in group2) 6-8 weeks after revascularization of stenosis

\section{Recording and Preprocessing}

- $10 \mathrm{~min}$. resting state 63 -channel EEG $(1000 \mathrm{~Hz}$ sampling frequency)

- Segmentation in $4 \mathrm{sec}$ windows

- Band pass filter 2- $256 \mathrm{~Hz}$; band stop at nyquist frequency

- Artifact rejection and ICA to reject eye movements

- Common average reference
EEG Source Reconstruction and Coherence Analysis

- BEM forward solution on individual T1 MRI

- Calculation of leadfields and beamformer (LCMV) in carotid artery network

- Project time series into source space

- Spectral analysis from $1-30 \mathrm{~Hz}$ (steps of $2 \mathrm{~Hz}$ )

- Calculation of the imaginary coherence $10 \times 10$ matrix

- Flip stenotic side to the right in all patients

- Average data in the alpha $(8-13 \mathrm{~Hz})$ and beta $(14-25 \mathrm{~Hz})$ band
Statistical Analysis

- Linear mixed model analysis using R/Ime4 3 models for:

group1 vs group2 group2 vs group3 group1 vs group3

Relationship between the connectivity (imaginary coherence) and groups with interaction of group and side

coherence $=\operatorname{Imer}($ group $*$ side $+(1 /$ id $)+$ (1/connections) $+(1 /$ frequency $))$ 